\title{
Retinal Blood Vessel Segmentation Using Gabor Wavelet and Line Operator
}

\author{
Reza Kharghanian and Alireza Ahmadyfard
}

\begin{abstract}
In this paper, we propose a method for segmenting blood vessels from retinal images. We extract two sets of features for image classification: features based on Gabor wavelet and line operator. At each pixel of retinal image we construct a feature vector consisting of the pixel intensity, four features from Gabor wavelet transform in different scales and two features from orthogonal line operators. We compare the result of classification using two classifiers: Bayesian and SVM. First we estimate class-conditional probability density functions for vessel and non-vessel using Gaussian mixture model. Then using a Bayesian classifier we implement a fast classification. The result of experiments show the combination of Gabor features and line features provides a good performance for vessel segmentation. We tested the proposed algorithm on DRIVE database which is publicly available. As the second classifier we employ Support Vector Machine. The results shows SVM classifier in some cases performs better than Bayesian classifier.
\end{abstract}

Index Terms-Retinal image, vessel segmentation, Gabor wavelet, line detector, supervised classification.

\section{INTRODUCTION}

Assessment of the characteristics of vessels plays an important role in a variety of medical diagnoses. For these tasks measurements of vessel width, color, reflectivity, tortuosity and abnormal branching are needed.

Previous methods for vessel segmentation in images of the retina can be divided into two groups. The first group consists of rule-based methods. The second group consists of supervised methods, which require manually labeled images for training.

Interesting results have been obtained by pixel classification based on supervised learning. The goal of classification approaches is to assign each pixel to one of two classes, namely vessel and non-vessel, based on some features extracted from the image in the neighborhoods of the considered pixel.

In [1], a method based on multi-scale Gabor analysis of retinal image was proposed. A feature vector consist of five features was used for vessel segmentation. At each pixel the grey level of the inverted green channel and the response of Gabor transform for four different scales are used as features. At each scale the maximum response of Gabor wavelet over different orientations spanning from $0^{\circ}$ to $179^{\circ}$ at step of $10^{\circ}$ is calculated. Image pixels in this method are classified using Bayesian classifier.

In [2], Ricci et al. use only three features for pixel

Manuscript received June 26, revised July 30, 2012.

The authors are with the Electrical and robotic engineering, Shahrood university of technology Shahrood, Iran (e-mail: r_kharghanian@yahoo.com; ahmadyfard@shahroodut.ac.ir). classification: the grey level of the inverted green channel image and response of two line detectors to the neighborhood of the pixel, one perpendicular to another. The basic line detector has length 15 pixels which rotate at 12 different orientations between 0 to 360 degrees. The response of line operator at each pixel along a specific angle is obtained by averaging the grey level of pixels along the line operator. Then the largest response is one of two line features. The average grey level of line with length equal to three pixels orthogonal to the basic line detector is used as another line feature. A linear support vector machine (SVM) [3] is employed as a classifier for segmenting vessels in retinal image.

In [4] a first-order derivative filters, known as difference of offset Gaussians filters (DoOG filters) is used, with prevailing responses to horizontal, vertical, and diagonal directions. These filters are used for the computation of the local image gradient in a specific direction. Then each of the four directional images resulting from the DoOG filters is searched for specific combinations of signs to extract vessel centerline. Vessel reconstruction phase includes multiscale TopHat filtering and a binary reconstruction.

The main problem in the above segmentation methods is the failure for detecting thin vessels as well as vessels in low contrast regions.

In this paper we proposed a method for classification of pixels in retinal image to extract vessels. We use a feature vector consist of seven components: the grey level of green channel, response of Gabor filter bank [1] (four scales) and the response of two line operators. We use Gaussian mixture model to estimate class conditional density of vessels and non-vessels. We use Bayesian classifier to classify pixels in retinal image.

The rest of the paper organized as follows. In section 2, the proposed method is described. The experimental results are presented in Section 3.

\section{THEORY}

\section{A. Feature Extraction}

We describe each pixel using a feature vector consist of seven features: the grey level of the inverted green channel image, the maximum Gabor transform response over angels at four different scales and the response of two orthogonal line operators.

\section{1) Inverted green channel}

The analysis of RGB components of retinal images shows the green channel has the best vessel/background contrast, whereas the red and blue channels tend to be very noisy. Therefore, the inverted green channel in which the vessels 
appear brighter than the background is used as input image to our vessel detection system. An iterative algorithm as proposed in [1] is used to remove the strong contrast between the retinal fundus and the region outside the aperture. This

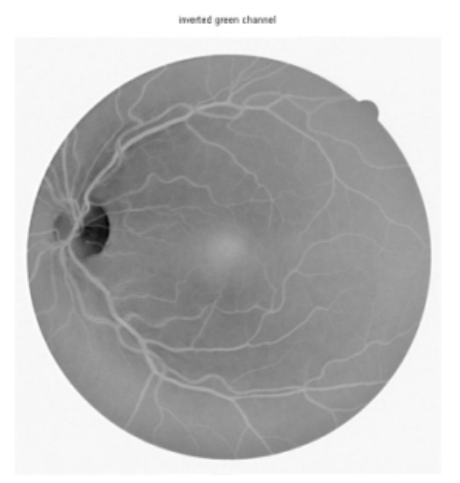

improves the undesired response of both the wavelet transform and the line operators at border of retinal disk. Fig. 1 shows the result of extending image at border of retinal image.

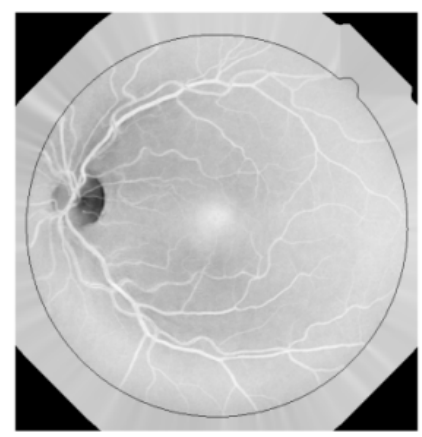

Fig. 1. (Left) inverted green channel of colored fundus image, (right) image with extended border

\section{2) Gabor wavelet features}

Let $f$ be an image defined on the real plane with finite energy and $\psi$ be the analyzing wavelet [5]. A family of wavelet can be defined by translations, rotations and dilations of the analyzing wavelet. The continuous wavelet transform is defined in terms of the scalar product of $f$ with the transformed wavelet [1], [6]:

$$
\begin{aligned}
T_{\psi}(\mathrm{b}, \theta, a)= & C_{\psi}^{-1 / 2}\left\langle\psi_{\mathrm{b}, \theta, a} \mid f\right\rangle \\
& C_{\psi}^{-1 / 2} a^{-1} \int \psi^{*}\left(a^{-1} r_{-\theta}(X-\mathrm{b})\right) f(X) d^{2} X
\end{aligned}
$$

Where $C_{\varphi}, \varphi$, b, $\theta$ and $a$ denote the normalizing constant, analyzing wavelet, the displacement vector, the rotation angle and the dilation parameter (also known as scale) respectively. The $\psi^{*}$ denotes the complex conjugate of $\psi$. The wavelet transform can be easily implemented using the fast Fourier transform algorithm and the equivalent Fourier definition of the wavelet transform [7]:

$$
T_{\psi}(\mathrm{b}, \theta, a)=C_{\psi}^{-1 / 2} a \int \exp (j \mathrm{~kb}) \hat{\psi}^{*}\left(a r_{-\theta} \mathrm{k}\right) \hat{f}(\mathrm{k}) d^{2} \mathrm{k}
$$

where $j=\sqrt{-1}$, and the hat denotes a Fourier transform. Since retinal blood vessels can appear in any direction, a directional filter has been chosen to prominent vessel patterns. 2-D Gabor wavelet has directional selectiveness capability of detecting oriented features and fine tuning to specific frequencies [7],[6]. This latter property is especially important in filtering out the background noise of the fundus images. The 2-D Gabor wavelet is defined as:

$$
\psi_{G}(X)=\exp \left(j \mathrm{k}_{0} X\right) \exp \left(-\frac{1}{2}|A X|^{2}\right)
$$

where $A=\operatorname{diag}\left[\varepsilon^{-1 / 2} 1\right], \varepsilon \geq 1$ is a $2 \times 2$ diagonal matrix that defines the anisotropy of the filter, i.e., its elongation in any desired direction. The Gabor wavelet is actually a complex exponential modulated Gaussian, where $k_{0}$ is a vector that defines the frequency of the complex exponential.

At a specific scale the maximum modulus of the wavelet transform over all angles, from $0^{\circ}$ to $179^{\circ}$ at step of $10^{\circ}$ steps is selected:

$$
M_{\psi}(\mathrm{b}, a)=\max _{\theta}\left|T_{\psi}(\mathrm{b}, \theta, a)\right|
$$

Using this process at each pixel four Gabor features is provided. Each feature is the response of Gabor filter in a specific scale.

\section{B. Line Operators}

At each pixel of image we extract two features using orthogonal line operators. The basic line operator is a line with length $l$ [2] centered at considered pixel. At each pixel the average of image gray level along line operators with 12 different orientations spanning 360 degrees are evaluated. The direction for which line operator provides the maximum gray level is selected and the corresponding gray level is denoted by $L$. This value is compared with average gray level of image, $\mathrm{N}$, within a square window centered at the pixel. The difference $S=L-N$ is used as a feature which measures the potential of the pixel being on a vessel [8].

The second feature of line operator is evaluated using gray level of the pixel neighborhood along the line perpendicular to the line operator of the first feature. The second line has three pixels length centered at the midpoint of the basic line operator and orthogonal to it. Its average value is denoted by $L_{0}$ and its strength is obtained by $S_{0}=L_{0}-N$. In fact for a pixel on vessel this value must be relatively large.

When a pixel is located on the background or thin vessels with low contrast, feature $L$ for both cases would be close, but feature $L_{0}$ extracted using orthogonal line operator can discriminates between the two cases. $L_{0}$ would be negligible for pixels on background while relatively large for pixels on thin vessels.

\section{Feature Normalization}

Accordingly each pixel of image is described by a feature vector with seven components. In the next step we normalize feature vector so that each feature has zero mean and unit 
standard deviations [9]:

$$
\widehat{v}_{i}=\frac{v_{i}-\mu_{i}}{\sigma_{i}}
$$

where $v_{i}$ is the ith feature assumed by each pixel, $\mu_{i}$ is the average value of the ith feature, and $\sigma_{i}$ is the standard deviation of associated feature.

Features extracted from each image must be normalized separately in order to compensate the inherent variation among images.

\section{D.Supervised Classification}

Several classifiers have been used for classification of pixels in a retinal image. In [1] a Bayesian classifier in [10], [11] and [12] a K-Nearest Neighbor and in [2] Support Vector Machine have been used as classifiers for pixel segmentation. In classification process each pixel of retinal image takes one of two labels: vessel or non-vessel. Bayesian and K-Nearest Neighbor require a large number of training pixels for design of classifier. In compare, the training of a Support Vector Machine requires less data. In this paper we used Bayesian and SVM classifiers separately to classify pixels in retinal images. For estimation of class conditional densities for Bayesian classifier we use Gaussian Mixture Model in Matlab. The SVM classifier has been implemented using SVMlight libraries [3], [13].

\section{EXPERIMENTAL RESULTS}

\section{A. Experimental Evaluation}

We tested the proposed method on database DRIVE [11]. The DRIVE database consists of 40 images (seven of which present pathology), along with manual segmentations of the vessels. The images are captured in digital form from a Canon CR5 nonmydriatic 3CCD camera at $45^{\circ}$ field of view (FOV). The images are of size $768 \times 584$ pixels, eight bits per color channel and have a FOV of approximately 540 pixels in diameter. The 40 images have been divided into training and test set, each containing 20 images (the training set has three images with pathology). They have been manually segmented by three observers trained by an ophthalmologist. The images in the training set were segmented once, while images in the test set were segmented twice, resulting in sets A and B. The observers of sets A and B produced similar segmentations. In set A, $12.7 \%$ of pixels where marked as vessel, against $12.3 \%$ vessel for set $B$.

In feature extraction step the inverted green channel of colored retinal image is used as the input image. Gabor wavelet transform applied in four scales 2, 3, 4 and 5; furthermore, we set the ${ }^{\varepsilon}$ parameter in the Gabor function to 4 , making the filter elongated and $k_{0}=\left[\begin{array}{ll}0 & 3\end{array}\right]$, i.e., a low-frequency complex exponential with few significant oscillations perpendicular to the large axis of the wavelet [1]. These two characteristics are specially suited for the detection of directional features and have been chosen in order to enable the transform to present stronger responses for pixels associated with the blood vessels. Basic line detector has used with $l=15$ pixels length [2].

In the first experiment 700,000 pixel samples were randomly chosen from 20 labeled training images to estimate density function of vessel and non vessel using GMM. The number of Gaussians in the mixture set to 20. In compare we used only 20,000 pixels from the huge number of training samples for finding the support vectors in SVM classifier.

In order to evaluate how discriminative are features in the feature vector we designed the second experiment. In this experiment each classifier has been designed using three different feature vectors. In the first set the feature vector contains three components: inverted green channel and the response of two line operators. In the second set a feature vector has 5 elements: inverted green channel, Gabor wavelet response for 4 scales. Finally in the third set feature vector augments the feature vectors in two previous cases including seven features.

\section{B. Results}

We tested the proposed method on the DRIVE database. The performance has been measured based on the segmentations of set $\mathrm{A}$ as ground truth. The segmentations of set $\mathrm{B}$ are tested against those of $\mathrm{A}$, serving as a human observer reference for performance comparison. We have selected accuracy criteria to facilitate the comparison with other retinal vessel segmentation algorithms. We define classification accuracy as the ratio of the number of correctly classified pixels by the total number of pixels in the image.

Table I shows the accuracy for different methods. In this table the proposed method has been compared with Soares et al. [1] and Ricci et al. [2] methods.

TABLE I: ACCURACY MEASUREMENT FOR DIFFERENT METHODS
\begin{tabular}{|l|c|c|c|}
\hline \multirow{2}{*}{ Method } & \multicolumn{3}{|c|}{ ACC } \\
\cline { 2 - 4 } & Min & Max & Avg. \\
\hline $\begin{array}{l}\text { Soares } \text { et al.(DRIVE) [1], } \\
\text { GMM \& K=20 }\end{array}$ & $\begin{array}{c}0.934 \\
1\end{array}$ & $\begin{array}{c}0.959 \\
5\end{array}$ & $\begin{array}{c}0.946 \\
0\end{array}$ \\
\hline Ricci et al.(DRIVE) [2], & 0.929 & 0.959 & 0.942 \\
SVM \& $l=15$ & 0 & 7 & 8 \\
\hline $\begin{array}{l}\text { Proposed } \\
\text { SVM \& 7 features }\end{array}$ & 0.935 & 0.963 & 0.946 \\
9 & 9 & 9 \\
\hline $\begin{array}{l}\text { Proposed } \\
\text { GMM with K=20 \& 7 features }\end{array}$ & 2 & 7 & 7 \\
\hline
\end{tabular}

As the result shows the combination of Gabor features and line features improves the performance. We observed the result of image classification using [1] and [2] to find the reason for method.

In [2], those pixels located between two thick vessels are misclassified as vessel while they belong to non vessel class (Fig. 2).

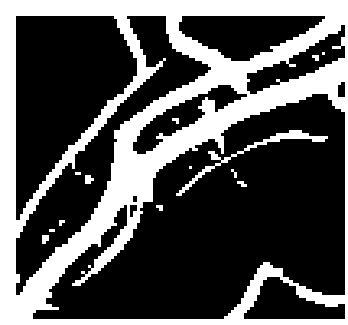

Fig. 2. Misclassification for pixels between thick vessels 
The proposed method in [1] fails in presence of the central reflex. This is observed on wide vessels as a bright strip along the center line. In Fig. 3 demonstrates this effect on segmentation result using the method in [1].

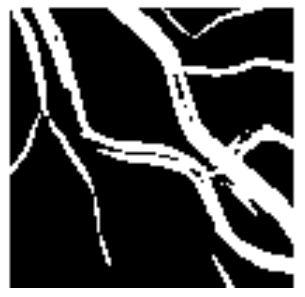

Fig. 3. Misclassification for central reflex

Also when two thick vessels are close, the method [1] fails to separate them (shown in Fig. 4).

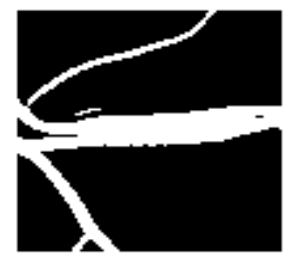

Fig. 4. Two thick vessels have been jointed

In some cases both methods in [1] and [2] fail. None of these methods are capable to segment the optic disk region and thin vessels properly. Combining the Gabor and line operator features improves the segmentation result.

In the second experiment we use different feature vectors for classification. Table II shows the result of this experiment. The result of vessel segmentation for two images from DRIVE database are shown in Fig. 5.

TABLE II: RESULT OF OUR EXPERIMENT

\begin{tabular}{|c|c|c|c|}
\hline \multirow{2}{*}{ Classifier } & \multicolumn{3}{|c|}{ ACC } \\
\hline & Min & Max & Avg. \\
\hline Bayesian, GMM, k=20, 3features & 0.9328 & 0.9618 & 0.9494 \\
\hline Bayesian, GMM, k=20, 5features & 0.9341 & 0.9595 & 0.9460 \\
\hline Bayesian, GMM, k=20, 7features & 0.9362 & 0.9617 & 0.9477 \\
\hline SVM, 3features & 0.9290 & 0.9597 & 0.9428 \\
\hline SVM, 5features & 0.9361 & 0.9583 & 0.9438 \\
\hline SVM, 7features & 0.9352 & 0.9639 & 0.9469 \\
\hline $\begin{array}{l}7 \text { features: intensity }+4 \text { Gabor output }+ \\
5 \text { features: intensity }+4 \text { Gabor output } \\
3 \text { features: intensity }+2 \text { line operator }\end{array}$ & & & \\
\hline
\end{tabular}
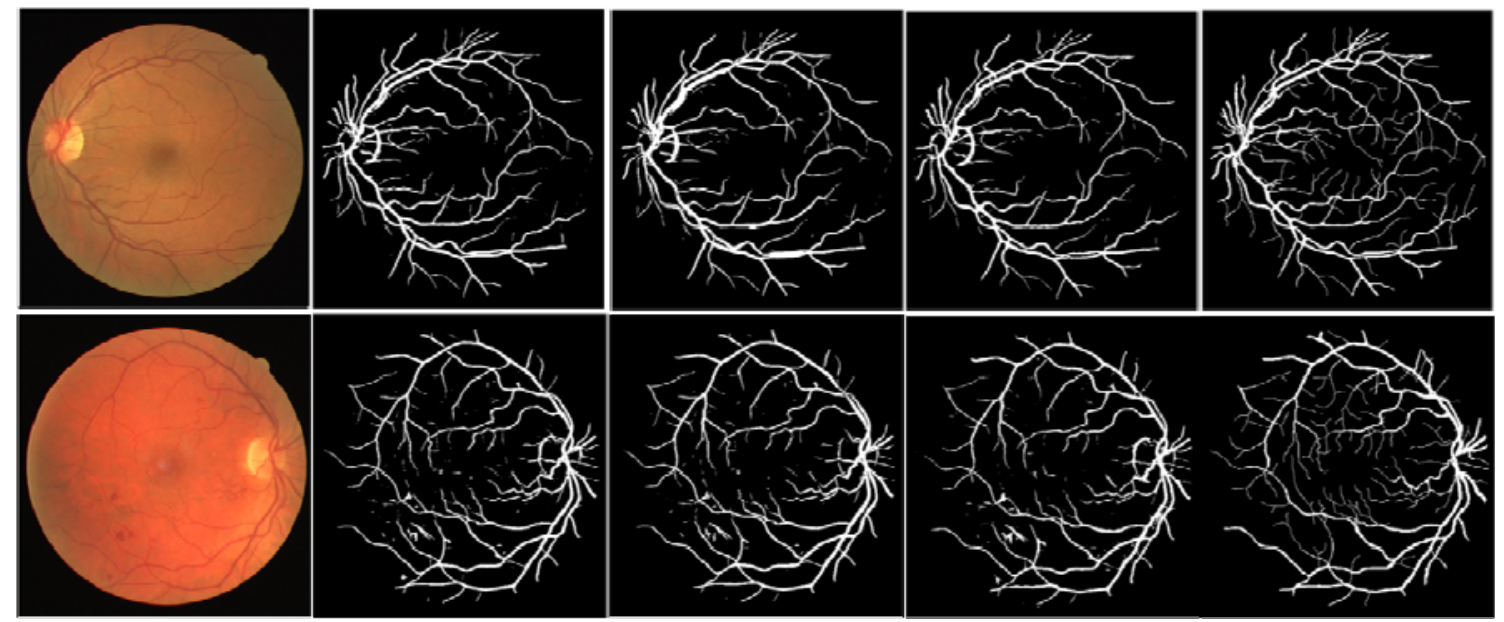

Fig. 5. The result of segmentation from the left: first column is input images. The second, third and forth columns are results of segmentation using 3 , 5 and 7 features respectively. The last column is manual segmentation by specialist.

Final vessel segmentation results for two images from DRIVE database along with the groundtruth are shown in Fig. 8. From the left, first column shows the colored image and second, third and forth columns are shown segmentation results using 3, 5 and 7 features respectively and the last column is shown the groundtruth of colored image.

\section{CONCLUSION}

In this paper a method for segmenting vessels in the retinal image was proposed. From each pixel a set of features using Gabor analysis and line operator are extracted. Using the extracted feature vector the classification was performed using Bayesian and SVM classifier. Very promising result for vessel segmentation was provided.

\section{REFERENCES}

[1] J. V. B. Soares, J. J. G. Leandro, R. M. Cesar, Jr., H. F. Jelinek, and M.J. Cree, "Retinal vessel segmentation using the 2D Gabor wavelet and supervised classification," IEEE Trans. Med. Image., vol. 25, no. 9, pp.1214-1222, Sep. 2006.

[2] E. Ricci and R. Perfetti, "Retinal blood vessel segmentation using line operators and support vector classification,” IEEE Trans. Med. Image., vol. 26, no. 10, Oct. 2007.

[3] J.-P. Antoine, R. Murenzi, P. Vandergheynst and S. Twareque Ali, Two-Dimensional Wavelets and their Relatives, Cambridge University Press, 2004.

[4] J. P. Antoine, P. Carette, R. Murenzi, and B. Piette, "Image analysis with two-dimensional continuous wavelet transform," Signal Process.,vol. 31, pp. 241-272, 1993.

[5] Nancy M.Salem and Asoke K. Nandi, "Segmentation of Retinal Blood Vessels Using Scale Space Features and K-Nearest Neighbor Classifier,” IEEE Conf 2006. 
[6] A. Arnéodo, N. Decoster, and S. G. Roux, "A wavelet-based method for multifractal image analysis. I. Methodology and test applications on isotropic and anisotropic random rough surfaces,” Eur. Phys. J. A, vol. 15, pp. 567-600, 2000.

[7] J. J. Staal, M. D. Abràmoff, M. Niemeijer, M. A. Viergever, and B. van Ginneken, "Ridge based vessel segmentation in color images of the retina," IEEE Trans. Med. Imag., vol. 23, no. 4, pp. 501-509, Apr. 2004.

[8] R. Zwiggelaar, S.M. Astley, C. R. M. Boggis, and C. J. Taylor, "Linear structures in mammographic images: Detection and classification," IEEE Trans. Med. Image., vol. 23, no. 9, pp. 1077-1086, Sep. 2004.

[9] L. F. Costa and R. M. Cesar-Jr, Shape Analysis and Classification:Theory and Practice. Boca Raton, FL: CRC, 2001.
[10] Leandro, J. J. G., Soares, J. V. B., Cesar, R. M., Jr., and Jelinek, H. F., "Blood Vssels Segmentation in Non-Mydriatic Images using Wavelets and Statistical Classifiers,". IEEE Computer Graphics and Image Processing, 2003. SIBGRAPI 2003.

[11] Software, [Online]. Available: http://svmlight.joachims.org/.

[12] A. M. Mendonça and A. Campilho, "Segmentation of retinal blood vessels by combining the detection of centerlines and morphological reconstruction," IEEE Trans. Med. Image., vol. 25, no. 9, pp. 1200-1213,Sep. 2006.

[13] T. Joachims, "Making large-scale SVM learning practical," in Advances in Kernel Methods, Support Vector Learning, B. Scholkopf, C.Burges, and A. Smola, Eds. Cambridge, MA: MIT Press, 1999. 